Published as: Epilepsy Behav. 2014 December ; 0: 183-192.

\title{
Proceedings of the Fifth International Workshop on Advances in Electrocorticography
}

\author{
Anthony Ritaccio ${ }^{a}$, Peter Brunner ${ }^{a, b}$, Aysegul Gunduz ${ }^{c}$, Dora Hermes ${ }^{d}$, Lawrence J. \\ Hirsch $^{\mathrm{e}}$, Joshua Jacobs ${ }^{\dagger}$, Kyousuke Kamada $^{\mathrm{g}}$, Sabine Kastner ${ }^{\mathrm{h}}$, Robert T. Knight ${ }^{i}$, Ronald \\ P. Lesser ${ }^{j}$, Kai Miller ${ }^{d}$, Terrence Sejnowski ${ }^{k}$, Gregory Worrell', and Gerwin Schalk ${ }^{a, b}$ \\ aAlbany Medical College, Albany, NY, USA \\ bWadsworth Center, New York State Department of Health, Albany, NY, USA \\ cUniversity of Florida, Gainesville, FL, USA \\ dStanford University, Stanford, CA, USA \\ eYale University School of Medicine, New Haven, CT, USA \\ fDrexel University, Philadelphia, PA, USA \\ gAsahikawa Medical University, Asahikawa, Hokkaido, Japan \\ hPrinceton University, Princeton, NJ, USA \\ 'University of California, Berkeley, CA, USA \\ jJohns Hopkins University School of Medicine, Baltimore, MD, USA \\ kSalk Institute for Biological Sciences, La Jolla, CA, USA \\ 'Mayo Clinic, Rochester, MN, USA
}

\section{Abstract}

The Fifth International Workshop on Advances in Electrocorticography convened in San Diego, CA, on November 7-8, 2013. In the interval year since the last workshop, advancements in methodology, implementation, and commercialization across both research and clinical interests were the focus of the gathering. Electrocorticography $(\mathrm{ECoG})$ is now firmly established as a preferred signal source for advanced research in functional, cognitive, and neuroprosthetic domains. Published output in ECoG fields has increased tenfold in the past decade. This proceedings attempts to summarize the state of the art.

(C) 2014 Elsevier Inc. All rights reserved.

Corresponding author: Anthony Ritaccio, MD, FAAN, FANA, Department of Neurology, Albany Medical College, Albany, NY, USA, RitaccA@mail.amc.edu, Tel: (518) 262-0554, Fax: (518) 262-6261.

This is a PDF file of an unedited manuscript that has been accepted for publication. As a service to our customers we are providing this early version of the manuscript. The manuscript will undergo copyediting, typesetting, and review of the resulting proof before it is published in its final citable form. Please note that during the production process errors may be discovered which could affect the content, and all legal disclaimers that apply to the journal pertain.

Conflict of Interest

We confirm that there are no known conflicts of interest associated with this publication and that there has been no significant financial support for this work that could have influenced its outcome. 


\section{Keywords}

electrocorticography; brain-computer interface; high-frequency oscillations; brain mapping; seizure detection; gamma-frequency electroencephalography; neuroprosthetics; subdural grid; functional mapping; electrical stimulation mapping

\section{Introduction}

\section{A. Ritaccio}

The Fifth International Workshop on Advances in Electrocorticography (ECoG) took place November 7-8, 2013, in San Diego, California. Advances in ECoG research and applications demand at least a yearly reckoning to keep pace with pertinent developments. This workshop has provided a venue for such a review since our inaugural meeting in 2008. This fifth workshop manifested the promise of ECoG-based recording by elucidating the evolution of stable clinical and research applications in the clinical, behavioral, and experimental neurosciences. Once again, strong emphasis was given to the role and contribution of the epilepsy patient as the window to human ECoG. Advances in methodology, implementation, and commercialization of passive ECoG functional mapping were updated. ECoG-driven neurobehavioral insights into frontal lobe functions, such as language prediction, were presented. A contemporary review of consensus and controversy in the recording and utility of pathologic high-frequency oscillations put a decade's worth of observations into practical perspective. The inevitable and valuable incorporation of ECoG tools in multimodal functional localization was summarized. Transformative changes in design and manufacture of high-density customized ECoG electrode arrays were described to an audience of clinicians and researchers expectant of improved stability and spatial resolution.

Neurostimulation and neuromodulation of brain functions have caught the attention of the media and the general public. The number of centers engaged in human ECoG-based research has proliferated tenfold in the past decade, as has publication volume (Fig. 1). United States government funding projects directed at ECoG-based researchers regularly constitute headline news [1]. At the same time, criticisms of underrepresented limitations and exaggerated claims within many neuroscientific disciplines, including ECoG, have also proliferated [2]. The earnest goal of these workshops and their accompanying proceedings is to provide a lucid, content-rich summary of the field to date, free from hyperbole.

\section{Clinical}

\subsection{Clinical Primer}

L.J. Hirsch—Intracranial electroencephalogram (EEG) recordings are indicated for the surgical treatment of refractory epilepsy when other tests to identify the seizure focus are discordant or inconclusive, when there is no magnetic resonance imaging (MRI) abnormality (except select medial temporal cases), when the seizure onset zone abuts eloquent cortex (including many lesional cases), and when there is dual pathology (e.g., hippocampal sclerosis plus a lesion). Although some cortical mapping and identification of 
the irritative ("spiking") zone can be done via brief intraoperative ECoG, implanted electrodes are usually required in order to identify the seizure onset zone. Complete removal of the seizure onset zone is associated with a greater chance of seizure freedom, even after accounting for lesion resection [3].

There is no good evidence that intraoperative ECoG can help guide neocortical resection during temporal lobectomy in patients with mesial temporal sclerosis; one study suggested it may be beneficial in guiding the posterior extent of hippocampal resection. Intraoperative ECoG may be adequate to guide resection in select cases with focal cortical dysplasia if continuous spiking is seen, as occurs in about two-thirds of cases [4]. There is no proven use for activation techniques, determination of afterdischarge thresholds, or elicitation of habitual auras/seizures in surgical planning, and there is some evidence that all of these can be misleading.

Complications of implanted intracranial electrodes occur in about $9 \%$ of patients and are mostly transient, with permanent deficits in $<2 \%$ and rare mortality [5]. Risks are higher with greater numbers of implanted electrodes, larger subdural grids, and peri-Rolandic location. The relative utility of subdural strips/grids, depth electrodes, stereo-EEG, and combinations of these is unknown. Recent gamma activation mapping techniques may speed up functional mapping, either intra- or extraoperatively [6].

Mesial temporal onset seizures on depth electrode recordings often begin with rhythmic spiking at $<2 \mathrm{~Hz}$ or low-voltage $10-$ to $16-\mathrm{Hz}$ activity. Unfortunately, spread to the hippocampus can look identical. Well-localized neocortical onsets often start with lowamplitude fast activity, typically $>16 \mathrm{~Hz}$, often $>30 \mathrm{~Hz}$. Many seizure onsets are difficult to localize to a discrete area and may involve wider epileptogenic networks, such as the limbic network, the occipital-lateral temporal network, and the parietal-frontal network [7].

High-frequency oscillations (HFOs; ripples: $80-250 \mathrm{~Hz}$, and fast ripples: $250-600 \mathrm{~Hz}$ ) may help localize epileptogenic tissue [8,9]. Fast ripples seem to be more specific than ripples for seizure onset zones, especially when associated with interictal spikes [10]. High-frequency oscillations may be more localizing than traditional interictal epileptiform discharges [8,9]. Identification of HFOs requires high sampling rates (preferably >2000 Hz) and different filter and "paper speed" settings or automated detection. One small recent study suggested that single-pulse stimulation-induced fast ripples were suggestive of the epileptogenic zone [11].

Devices are now available for recording chronic ambulatory intracranial EEG. Such devices may allow seizure prediction and warning, which would improve patient safety and quality of life, as well as allow responsive treatment for seizure prevention (e.g., via stimulationnow FDA approved, cooling or medications). Electrocorticographic signal analysis is also useful for brain-computer interfaces.

(Section 2.1 was presented at the Fourth International Workshop on Advances in Electrocorticography [12] and is reprinted with permission.) 


\subsection{What is Testing the Brain Telling Us?}

R.P. Lesser-Electrical stimulation was described as a treatment for disease at the time of the Roman empire and as a treatment for epilepsy in 13th century Persia. In the 18th century, Benjamin Franklin studied electrical stimulation using Leyden jars, and his studies included at least one person with a history of seizures. Wilder Penfield and collaborators used stimulation to define functional brain areas. On the basis of their work, and that of others, standard homunculi are often drawn suggesting a set location for all body representations along the sensorimotor strip. Although sensorimotor locations in general conform to those shown on homunculi, there are frequent deviations from the set patterns implied by these illustrations. There can be multiple widespread sites for specific body parts; these sites can overlap, and their locations can vary over time.

One goal of seizure treatment is complete control of seizures, or at least of seizures interfering with consciousness. Because seizures continue in one-quarter to one-third of patients despite anticonvulsant medication, epileptologists often consider performing epilepsy surgery. However, standard surgical techniques may not be applicable due to difficulties in localization or potential consequences of operating in eloquent cortex. This in turn has led to interest in additional treatments such as stimulation. The vagus nerve stimulator and, more recently, deep brain stimulation (in Europe) and the responsive neurostimulation device (in the USA), have been approved as treatments for intractable epilepsy. Although they are used in patients whose seizures cannot be controlled by standard surgical techniques, their efficacy has been modest. Why is that?

Penfield and Jasper, when defining functional areas, found that stimulation could produce afterdischarges (ADs). These usually are an unwanted side effect of brain stimulation, but since they are, in effect, induced electrical seizure patterns, studying them may help us to understand how seizures occur and how to terminate them. Just as there are variations in the functional responses to stimulation, there are variations in the occurrences of ADs. Just as functional localizations can vary, stimulation at the same intensity does not necessarily produce ADs with repeated stimulation, and the sites to which ADs spread can vary from one stimulation event to the next $[13,14]$.

When stimulation through electrodes produces ADs, brief subsequent pulses of stimulation through the same electrodes can terminate the ADs, but this occurs only about half the time [15]. The likelihood of terminating ADs is better if stimulation begins at the negative peak of the $\mathrm{AD}$ waveform [16]. Beyond this, there are no specific stimulation parameters that have been shown to consistently improve the likelihood of terminating ADs [14]. If we understand more about the basic physiology, biochemistry, and microanatomy underlying the responses to stimulation, we will be more likely to improve the efficacy of stimulation for treating seizures.

\subsection{Mapping Pathological Oscillations in Human Epileptogenic Brain}

G.A. Worrell-Successful localization of the epileptogenic networks generating spontaneous seizures is critical for successful epilepsy surgery and therapeutic focal brain stimulation. Recording spontaneous seizures with intracranial EEG (iEEG) remains the gold 
standard for localizing epileptogenic brain. Conventional clinical iEEG, however, records brain activity over relatively coarse spatial (approximately centimeter) and spectral ( $\sim 0.5-$ $100 \mathrm{~Hz}$ ) scales. Emerging evidence from multiple laboratories reports that current clinical iEEG is not optimal for localizing the epileptogenic zone (EZ), i.e., the pathological tissue that must be resected for long-term seizure freedom.

There is a critical need for refining EZ localization in order to improve epilepsy surgery and focal brain stimulation outcomes. Electrophysiological biomarkers (EB) are defined as objectively measured signals that can differentiate normal from pathological tissue $[17,18]$. Advances in human brain electrophysiology have led to the discovery of multiple EB [1922] that show potential for improving EZ localization. One of the most promising biomarkers of the EZ is pathological HFO (for recent reviews, see Engel [17] and Worrell and Gotman [18]).

Classification of physiological HFO and pathological HFO remains a central challenge for modern epileptology. High-frequency oscillations spanning gamma $(30-100 \mathrm{~Hz})$, ripple $(100-250 \mathrm{~Hz})$, and fast ripple $(250-600 \mathrm{~Hz})$ frequency oscillations are associated with normal neurophysiology (for recent review, see Buzsáki and Silva [23]). In brain capable of generating spontaneous seizures, however, interictal gamma [20], ripple [24], and fast ripple [19] HFOs are increased. While distinguishing between epileptiform HFOs and physiological HFOs remains a challenge, there has been recent progress [25]. There are multiple potential clinical applications if reliable interictal EB of epileptogenic brain can be established. Intraoperative recordings and interictal EB mapping could be used to improve intracranial electrode positioning and might even eliminate the need for prolonged iEEG monitoring required to record spontaneous seizures. The ability to map the EZ with intraoperative recordings would transform epilepsy surgery by reducing patient discomfort, risk, cost, and duration of presurgical evaluation.

\subsection{Mapping Cortical Function at the Bedside: Active}

A. Ritaccio-Electrical stimulation mapping (ESM) remains a pivotal method in clinical neurology and systems neuroscience. The interest and fascination with direct cortical electrical stimulation parallels the earliest awareness of the electrical nature of neural transmission.

The historical and clinical relevance of ESM are its best-evolved aspects. Giovanni Aldini (1756-1826), nephew of Luigi Galvani, used Volta's bimetallic pile to apply electric current to "reanimate" dismembered bodies of animals and humans (Fig. 2). In doing so, he became the primogeniture of generations of physiologists and neuroscientists to follow, including Rolando, Fritsch and Hitzig, Ferrier, Cushing, Krause, and Penfield. His experiments were also Mary Shelley's main inspiration in the creation of her 1818 novel, Frankenstein [26]: "Perhaps, a corpse would be reanimated; galvanism had given token of such things."

Observed behavioral phenomena induced by ESM have been in the realm of cessation of an ongoing behavior (e.g., speech arrest), simple motor behavior, illusions (i.e., distortion of sensory stimuli mostly in visual or auditory domains), recall of past events, emotional 
feelings, and hallucinations (i.e., induced perception in the absence of relevant sensory stimuli) [27].

The clinical utility of ESM in contemporary neurosurgery is greater than a half century in practice. Its utility is well established. Less than $2 \%$ of 250 patients sustained language deficits after resecting ESM-based language localization [28], and 87\% of 55 patients were without motor deficit in tumor resective surgery near ESM-defined motor regions [29]. In contrast, partial resection of ESM language sites commonly result in permanent linguistic deficit [30]. ESM is considered methodologically superior to diffusion tensor imaging (DTI) and functional magnetic resonance imaging (fMRI) in predicting tissue function in tumor surgery.

There has been a renewed interest in understanding ESM mechanisms and in distinguishing its well-established electrical diffusion effects (local) versus biologic diffusion (synaptic connectivity). Local effects, as predicted by a three-dimensional finite element model of current flow, demonstrate a sharp drop in current midway between electrodes commonly in clinical use [31]. However, it is both logically and experimentally clear that perceptual and behavioral phenomena induced by electrical charge to a brain region are likely due to change of activity in network of brain areas (including subcortical regions). The spatial extent of blood oxygen level-dependent (BOLD) changes induced by ESM suggests synaptic propagation through horizontal monosynaptic connections that mimic neural recruitment (electrical stimulation of $\mathrm{V} 1$ inducing BOLD changes in $\mathrm{V} 2, \mathrm{~V} 3$, and MT in a primate model) [32].

The best example of ESM-based distant modulation in humans is best exemplified by the methodology of cortico-cortical evoked potentials (CCEP) [33]. Cortico-cortical functional connectivity is elicited by stimulating a part of the brain through ECoG electrodes and recording evoked potentials that emanate from a distant region of the cortex via neuronal projections.

CCEP as a method to determine "probabilistic functional tractography" [34] holds great promise in advancing the contribution of ESM in the new millennium. Fine-tuning of this technique may give clinicians and researchers a potent tool to finally map networks and not nodes.

\subsection{Mapping Cortical Function at the Bedside: Passive}

P. Brunner-Mapping of cortical function is often performed prior to resective brain surgery. In this procedure, dysfunctional (e.g., cancerous or epileptogenic) cortex is delineated from areas that, if resected, would impair important functions of the patient. Currently, electrical cortical stimulation (ECS) remains the most widely accepted mapping technique. With this technique, pulses of electricity, e.g., 300- $\mu$ sec duration, 50 times per second, 1-15 mA current, are delivered directly to the cortex through a pair of electrodes. This stimulation can elicit inhibitory (e.g., for language) or excitatory (e.g., for motor) effects that can be observed in the patient's behavior. 
While ECS is well established, it does have inherent problems. For example, inadvertent current spread and afterdischarges can inhibit or excite cortical areas distant to the intended stimulation site. Although reducing the stimulation intensity can mitigate this undesired effect, effective mapping of some cortical functions (e.g., language) may require relatively high stimulation intensities. Additionally, mapping large cortical areas, with such higher stimulation currents, is not only time-consuming but can also cause adverse effects such as seizures and tissue damage.

The advent of passive functional brain imaging techniques such as fMRI, EEG, magnetoencephalography (MEG), and ECoG may provide new avenues to mapping cortical function. Rather than actively inducing or inhibiting function, these techniques correlate behavior with changes in the metabolic or electromagnetic brain activity. While such passive techniques can overcome some of the problems of ECS by identifying brain locations that exhibit task-related changes, they cannot yet determine whether all these locations are indeed necessary for a particular function. Despite this limitation, passive functional mapping, primarily using fMRI and ECoG, is now used in some clinics to guide ECS and thereby reduce the adverse effects of ECS.

In this context, the use of ECoG is very practical. This is mainly due to two reasons: First, placement of ECoG electrodes is already included in many resective brain surgery protocols (e.g., epilepsy surgery), or the placement does not add significant risk (such as in intraoperative scenarios). Second, the ECoG grid provides a directly visible coordinate system for the neurosurgeon, which removes the otherwise needed and error-prone coregistration of functional maps with the visible cortical anatomy. The unique utility of this approach has been demonstrated in several academic and clinical papers $[6,35,36]$ and recently led to the development and clinical validation of a commercially available passive ECoG-based mapping system called cortiQ [37]. While these recent developments are encouraging, the clinical utility of this new mapping technique for minimizing postsurgical deficits in different patient populations remains to be determined.

\section{Science}

\subsection{Tutorial on ECoG Physiology: the Spatial Distribution of Phase Coherence in the Movement-Related $\beta$-Rhythm}

K.J Miller, D. Hermes-ECoG potential changes in primary motor cortex during movement are dominated by broadband spectral increases and narrowband (oscillatory) decreases [38]. The broadband increases are a generic correlate of mean population firing rate $[39,40]$ and therefore are spatially focal, specific for individual movement modalities, and temporally precise [41-43]. $\beta$-Rhythm power decreases, in contrast, are spread over much larger regions of the brain, of which the functionally precise sites are a small subset.

These ECoG $\beta$-rhythm power decreases are seen with engagement of motor cortex during basic movements, movement imagery, or imagery-based online feedback $[38,40,42,44,45]$. They explain low-amplitude fMRI-BOLD signal changes outside of functionally specific motor regions, as identified by broadband ECoG or electrical stimulation [45]. $\beta$-Rhythm and broadband have fundamentally different physiologies. For example, a 19-year-old who 
suffered from a perinatal stroke was found to have distinct, independent, broadband representation of both hands on the healthy hemisphere. The $\beta$-rhythm representation, in contrast, was shared between both hands and favored the hand of the native representation [47].

The spatial extent of phase coherence in cortical brain rhythms is important: it can be used to directly address whether widespread power changes in a specific frequency range reflect a single physiological process that changes over a distributed brain area, or many processes that are similar in character and function but which are actually separate from one another. To determine the extent to which $\beta$-rhythms at neighboring cortical sites are generated by common versus independent sources, we examined the pairwise phase coherence of the $\beta$ rhythms across sites [43]. In contrast to many studies in which the absolute value of phase coherence (or mean-squared phase coherence) is used, we retain the phase-shift between sites. If there is a strong, coherent rhythm in a large fraction of the electrode array, then the act of re-referencing will introduce false phase coherence, $\pi$ out of phase. When this occurs, it can be revealed by examining the phase of the phase coherence. Figure 3 plots the phase coherence relative to a chosen reference site at which broadband power was maximally correlated with movement of the index finger. Figure 3 shows that phase coherence of the $\beta$ rhythm is spatially distributed over neighboring sites. Furthermore, by projecting each phase coherence value (i.e., each phase coherence vector in the complex plane) onto the strongest phase coherence value (i.e., a reference vector), we can measure the extent to which phases are aligned among the coherent sites. This projection procedure facilitates the identification of variation in phase across different cortical sites. The spatially coherent rhythms are gyrally delineated along the precentral and postcentral gyri. The phase coherence measure either decreases in magnitude or "skips" in phase as sulci are crossed. Thus the phase coherence motifs for the motor rhythm with M1 obey sulcal boundaries.

\subsection{Tutorial on ECoG Methodologies}

A. Gunduz-Historically, many of the earlier human ECoG studies were conducted by research-oriented clinicians due to their direct access to neurosurgical patients undergoing resective surgery for the treatment of intractable epilepsy [48,49]. Today, with the advancements in neural interfacing technology and the expanding neuroscientific literature, ECoG-based research is being increasingly conducted by multidisciplinary groups comprising clinicians, engineers, and neuroscientists. This section aims to provide the basic methodologies for those from all backgrounds interested in ECoG research. We also refer the readers to a video tutorial on ECoG signal acquisition and analysis provided in Hill et al. [50].

To avoid interference with clinical monitoring and for independence from clinical hardware or software filter settings, a bioamplifier system dedicated to research is highly recommended. Since time with patients is limited, it is good practice to set up robust and reliable recording hardware and software, which should be tested ahead of time and can be easily adapted to new patient-specific parameters. The clinical and research systems should have separate grounds so as not to interfere with each other. Both grounds should be chosen far away from predicted epileptic foci and from functional areas of interest for research. 
Since there will be interference within a hospital room, it is important to locate the sources of noise and eliminate them prior to the recording session in order to capture the highest quality signal possible. Most artifacts common across all channels (e.g., line noise) can be removed by spatial filtering, such as Laplacian or common average reference (CAR) filtering [51]. Artifacts isolated to a single or a few channels should be manually removed prior to spatial filtering to prevent spreading of artifacts to other channels.

ECoG signals exhibit broad spectral ranges that modulate with behavior. These spectral features can be identified by contrasting spectra during behavioral engagement with baseline spectra. These task-relevant modulations are mainly captured in the rhythmic oscillations (which appear as peaks in power spectra and are spatially broad observations) and asynchronous local activity (which appears as spatially localized broadband modulations over spectral ranges greater than $70 \mathrm{~Hz}$ ) [38]. Although these event-related desynchronizations and synchronizations have been known since the 1990s [44,52], only recent advancements in signal acquisition (which have enabled higher temporal resolution and signal-to-noise ratios) and computational technologies (which have enabled multidimensional signal processing and real-time applications) have facilitated the practical and widespread use of these ECoG features in human neuroscience and neural engineering.

Despite these advancements, the spatial resolution of recordings is still limited to the spacing of clinical grids (4-mm-diameter contacts with 1-cm spacing), and spatial coverage is limited to areas of clinical interest to minimize the risks associated with implants.

Although many research groups recognize the need for highly dense, biocompatible, and fully implantable ECoG grids, such technologies will be subjected to long approval processes before clinical use. Nevertheless, research and validation in these areas should be able to pave the way for adoption of these technologies in humans in the near future.

\subsection{Frontal Cortex and Cognition: Evidence from ECoG}

R.T. Knight-Electrocorticography has emerged as a powerful method to study human cognition across multiple domains. Neuropsychological and neuroimaging findings have shown that attention is supported by a distributed frontoparietal network that is altered in numerous neurological disorders. ECoG recording has delineated the precise neural mechanisms in humans supporting this ability to allocate attention. ECoG recording has revealed that the frontoparietal attention network is supported by three distinct electrophysiological mechanisms [53]. First, attended and correctly detected events generate a robust and sustained high gamma response. Second, coherence between frontal and parietal electrodes in the $2-5 \mathrm{~Hz}$ band increases for attended events. Finally, cross-frequency coupling between the low-frequency rhythm and high gamma is also increased for attended events. Crucially, the degree of cross-frequency coupling observed at the single-trial level predicts reaction time. These observations provide support for coherence as a method for engaging distributed networks in the service of cognition and highlight the key role of crossfrequency coupling in the tuning of local cortical regions during behavior. Cross-frequency coupling has been shown to support other aspects of human cognition. For instance, it has been shown that maintenance of multiple items in working memory is linked to the coupling of neural assemblies in the $25-100 \mathrm{~Hz}$ gamma range with theta phase in the hippocampus 
[54]. Furthermore, increasing the number of items (i.e., memory load) is associated with modulating this cross-frequency coupling by increasing the length of theta cycles to incorporate additional pockets of higher-frequency power increases. The role of cross frequency coupling extends to the motor domain where the degree of coupling in premotor and motor cortices precisely tracks motor learning across a range of tasks [55]. Similar findings have been reports in intracranial recordings from the nucleus accumbens, where cross-frequency coupling had been shown to index action monitoring during cognitive control [56]. These findings spanning attention, memory, motor learning, and cognitive control highlight the fundamental role of cross-frequency coupling in human cognition.

\subsection{Spatial and Temporal Dynamics of ECoG Related to Visual Stimuli}

K. Kamada-Recently, high gamma activity (HGA) has been shown to be positively correlated with BOLD responses in the primary cortical areas in animals and humans. It is, however, an open question whether similar relationships can be found in the association cortices. The aim of this study was to clarify spatial and temporal profiles of the HGA and BOLD in the language-related association cortices.

Thirteen patients with intractable epilepsy underwent fMRI and ECoG with word interpretation task to elicit language-related responses. Percent signal change of BOLD was calculated at the site of each ECoG electrode, and HGA of each electrode was obtained as a power amplification of high gamma band $(60-120 \mathrm{~Hz})$ activity. We made quantitative comparisons between them and described their spatial relationships on a template brain (Fig. 4).

HGAs were detected not only in the primary cortical areas but also in the language-related cortices such as the inferior frontal and middle temporal gyri. We demonstrated a positive correlation between HGAs and BOLD responses, suggesting that the HGAs could be physiological correlates of BOLD responses (Fig. 5).

The time-course analysis revealed different temporal dynamics of HGAs in the various regions. The frontal lobe showed longer-lasting HGA than the temporal lobe (Fig. 6).

In conclusion, we revealed a positive coupling of HGA and BOLD in association cortices using language task. Different temporal profiles of HGA in the frontal and temporal lobe might reflect the spatial dissociation of HGA and BOLD. This study contributes to extending the concept of HGA-BOLD coupling from primary to association cortices and will facilitate practical utilization of fMRI for evaluating higher-order cognitive functions not only in neuroscience researches but also in clinical application.

\subsection{The Electrophysiology of Human Memory Representations}

J. Jacobs-Electrocorticographic (ECoG) recordings are one of the most powerful tools available for human electrophysiology research. ECoG has been widely established as providing spatially and temporally precise information on how brain regions support particular behaviors. However, an underappreciated aspect of ECoG is that it also reveals the specific content of a neuronal network during memory and cognition [57]. Below, I explain 
how these content-specific ECoG signals elucidate specific neuronal patterns that underlie human memory.

As a person performs a memory task, neurons in various regions activate to represent the specific memory items that are encoded, maintained, and recalled. ECoG signals at particular frequencies and electrodes reveal many of these patterns by exhibiting variations in amplitude when patients view certain stimuli [58]. Figures 7A and 7B show an example of this phenomenon, depicting the ECoG activity of an electrode in ventral-temporal cortex that had increased high gamma $(65-150 \mathrm{~Hz})$ activity when the patient viewed letters $\mathrm{Q}$ or $\mathrm{L}$ in a memory task. Thus high-frequency ECoG signals can reveal the engagement of a neuronal network in supporting memory for certain items.

ECoG recordings measure neuronal activity at various frequencies simultaneously. Not only is content-specific information also present at multiple frequencies, but often there is independent information at separate bands. A recent study demonstrated this by showing that a multivariate algorithm that combined information across frequencies more accurately predicted the content of a patient's memory compared with a predictor that used even the best individual frequency band [59]. An important area of ongoing work is to differentiate the types of neural and cognitive information that are represented at separate frequencies.

In addition to ECoG showing the neuronal patterns that represent the content of memory, it can also explain how electrical brain stimulation affects cognition [60]. In one patient who underwent ECoG stimulation mapping, electrical stimulation at a particular electrode caused the patient to spontaneously recall memories of his high school (Fig. 7C). Subsequently, the patient performed a memory task where he recalled memories from several categories. Remembering information related to his high school caused a relative decrease in the amplitude of high gamma ECoG activity (Fig. 7D). This decreased activity appeared at the one electrode where stimulation caused him to recall high school memories.

The correspondence at this ECoG electrode between stimulation and high gamma signals is important theoretically because it helps explain how brain stimulation can initiate memory retrieval. In contrast to the view that brain stimulation randomly initiates memory retrieval [61], these data indicate stimulation can initiate "mental time travel" by artificially recreating a pattern of neuronal activity that resembles one found from normal cognition. Going forward, ECoG analyses that differentiate the specific content of a particular cognitive or behavioral task have the potential to elucidate new important details regarding the neural signals and computations that support behavior.

\subsection{Neural Mechanisms of Attention Control in the Primate Brain}

S. Kastner-Selective attention mechanisms route behaviorally relevant information through large-scale cortical networks. While there is evidence that populations of cortical neurons synchronize their activity to preferentially transmit information about attentional priorities, it is unclear how cortical synchrony across a network is accomplished. Based on its anatomic connectivity with the cortex, we hypothesized that the pulvinar, a thalamic nucleus, regulates cortical synchrony. To test this idea, we mapped pulvino-cortical networks within the visual system using diffusion tensor imaging and simultaneously 
recorded spikes and field potentials from these interconnected network sites in monkeys performing a visuo-spatial attention task. We have shown that the pulvinar synchronized activity between two interconnected cortical areas according to attentional allocation, suggesting not only a critical role for the thalamus in attentional selection, but a more general role in regulating information transmission across visual cortex.

\subsection{Interactions Between Core and Matrix Thalamocortical Projections in Human Sleep Spindle Synchronization}

T. Sejnowski-Sleep spindles are bursts of $11-15 \mathrm{~Hz}$ that occur during non-rapid eye movement sleep. Spindles are highly synchronous across the scalp in the EEG but have low spatial coherence and exhibit low correlation with the EEG when simultaneously measured in the MEG. We developed a computational model to explore the hypothesis that the spatial coherence spindles in the EEG is a consequence of diffuse matrix projections of the thalamus to layer 1 compared with the focal projections of the core pathway to layer 4 recorded in the MEG. Increasing the fan-out of thalamocortical connectivity in the matrix pathway, while keeping the core pathway fixed, led to increased synchrony of the spindle activity in the superficial cortical layers in the model. In agreement with cortical recordings, the latency for spindles to spread from the core to the matrix was independent of the thalamocortical fan-out but highly dependent on the probability of connections between cortical areas.

\subsection{Single-Trial Characterization of ECoG Visuomotor Networks}

G. Schalk-Behavior arises from neuronal population activity that occurs across distant areas of the brain. Charting this activity across space and time, and examining its relationship to different aspects of neurophysiology or behavior, is of central importance to advancing our understanding of brain function. Studies over the past decades have been successful in identifying the locations of neuronal populations involved in specific behaviors, or in characterizing the specific relationship of individual populations of neurons to task-related variables. They have only been marginally successful in characterizing the spatial and temporal sequence of distant populations of neurons that together implement a complex behavior, such as visuomotor function.

Because it has high spatial and temporal resolution, electrocorticography appears to be well suited to investigate this activity sequence. At the same time, the timing of cortical activity is known to vary from trial to trial. Thus, the activity sequence of such cortical activity cannot readily be derived by applying traditional analytic techniques, such as cross-trial averaging. Hence, it remains largely unknown how neuronal activity traverses space and time, and how it relates to behavior or to other physiological mechanisms such as modulatory oscillatory activity.

In our study, we set out to begin to address this critical problem. To do this, we recorded ECoG signals in four human subjects while they performed a visuomotor response task: subjects pressed a button as soon as they detected a change in a visual stimulus. We then applied a novel detection technique to identify the time of activity onset and offset in neuronal populations in single trials. The results show that population activity at each 
location was typically much briefer than that implied by previous studies. Furthermore, they demonstrate that variance in the timing of population activity is strongly related to variance in the timing of the behavior. Finally, our results reveal an intriguing preference of the timing of the onset of cortical activity to the first half of the trough of underlying oscillatory activity in the alpha band. Our results document this effect across all subjects and across many brain regions.

In summary, our recent methodological advances allow us to chart the spatial and temporal progression of neural activity from its beginning in visual cortex to its end in sensorimotor cortex. Our initial results provide tantalizing evidence for a general physiological mechanism that governs the activation and/or synchronization of distant neural populations.

\section{Perspectives/Conclusion}

\section{G. Schalk}

Electrocorticography is the technique of interrogating the brain using electrodes that are placed subdurally or epidurally. Over the past decade, the important role of ECoG for basic research and its potential to create a new range of clinical applications has become increasingly recognized. Indeed, over the past few years, ECoG research articles have even succeeded to be accepted in the most renowned scientific journals, including Science and Nature.

The increasing recognition of the ECoG platform is encouraging. At the same time, the potential of ECoG for studying the brain and for using it to produce new diagnostic or therapeutic options is likely much greater than suggested by these initial encouraging demonstrations. Our methods for characterizing the complex task-related ECoG processes that unfold across space and time, and our ability for interacting with these processes for diagnostic or treatment purposes, are still rather primitive. Thus, with the further expected improvements in these areas, the value of ECoG for basic and translational neuroscience is likely going to continue to increase substantially.

\section{Acknowledgments}

This research was partially supported by the NIH [R37-NS21135 (R.T.K.), R01-EY017699 (S.K.), R21-EY023656 (S.K.), R01-NS065186 (K.J.M.), R01-EB000856 (G.S.), and R01-NS63039 (G.W.)], the U.S. Army Research Office [W911NF-08-1-0216 (G.S.), W911NF-12-1-0158 (G.S.), W911NF-12-1-0109 (G.S.), and W911NF-13-1-0479 (G.S.)], the Brain and Behavior Research Foundation (J.J.), the Fondazione Neurone (G.S.), and the Japanese Ministry of Education, Culture, Sports, Science and Technology [Grant-in-Aid for Scientific Research (B) No. 24390337, 2012-2015 (K.K.); and Grant-in-Aid for Exploratory Research No. 26670633, 2014 2016 (K.K.)].

\section{References}

1. Carey, B. Probing brain's depth, trying to aid memory. The New York Times Online; Jul 9. 2014 http://www.nytimes.com/2014/07/09/health/probing-brains-depth-trying-to-aid-memory.html [Accessed 25 July 2014]

2. Satel, S.; Lilienfeld, SO. Brainwashed: the Seductive Appeal of Mindless Neuroscience. New York: Basic Books; 2013. 
3. Asano E, Juhász C, Shah A, Sood S, Chugani HT. Role of subdural electrocorticography in prediction of long-term seizure outcome in epilepsy surgery. Brain. 2009; 132:1038-1047. [PubMed: 19286694]

4. Palmini A, Gambardella A, Andermann F, Dubeau F, da Costa JC, Olivier A, et al. Intrinsic epileptogenicity of human dysplastic cortex as suggested by corticography and surgical results. Ann Neurol. 1995; 37:476-487. [PubMed: 7717684]

5. Wong CH, Birkett J, Byth K, Dexter M, Somerville E, Gill D, et al. Risk factors for complications during intracranial electrode recording in presurgical evaluation of drug resistant partial epilepsy. Acta Neurochir (Wien). 2009; 151:37-50. [PubMed: 19129963]

6. Brunner P, Ritaccio AL, Lynch TM, Emrich JF, Wilson JA, Williams JC, et al. A practical procedure for real-time functional mapping of eloquent cortex using electrocorticographic signals in humans. Epilepsy Behav. 2009; 15:278-286. [PubMed: 19366638]

7. Spencer SS. Neural networks in human epilepsy: evidence of and implications for treatment. Epilepsia. 2002; 43:219-227. [PubMed: 11906505]

8. Jacobs J, Zijlmans M, Zelmann R, Chatillon CE, Hall J, Olivier A, et al. High-frequency electroencephalographic oscillations correlate with outcome of epilepsy surgery. Ann Neurol. 2010; 67:209-220. [PubMed: 20225281]

9. Wu JY, Sankar R, Lerner JT, Matsumoto JH, Vinters HV, Mathern GW. Removing interictal fast ripples on electrocorticography linked with seizure freedom in children. Neurology. 2010; 75:16861694. [PubMed: 20926787]

10. Wang S, Wang IZ, Bulacio JC, Mosher JC, Gonzalez-Martinez J, Alexopoulos AV, et al. Ripple classification helps to localize the seizure-onset zone in neocortical epilepsy. Epilepsia. 2013; 54:370-376. [PubMed: 23106394]

11. van 't Klooster MA, Zijlmans M, Leijten FS, Ferrier CH, van Putten MJ, Huiskamp GJ. Timefrequency analysis of single pulse electrical stimulation to assist delineation of epileptogenic cortex. Brain. 2011; 134:2855-2866. [PubMed: 21900209]

12. Ritaccio A, Brunner P, Crone NE, Gunduz A, Hirsch LJ, Kanwisher N, Litt B, Miller K, Moran D, Parvizi J, Ramsey N, Richner TJ, Tandon N, Williams J, Schalk G. Proceedings of the Fourth International Workshop on Advances in Electrocorticography. Epilepsy Behav. 2013; 29:259-268. [PubMed: 24034899]

13. Lesser RP, Lee HW, Webber WR, Prince B, Crone NE, Miglioretti DL. Short-term variations in response distribution to cortical stimulation. Brain. 2008; 131:1528-1539. [PubMed: 18337272]

14. Lesser RP, Crone NE, Webber WR. Subdural electrodes. Clin Neurophysiol. 2010; 121:13761392. [PubMed: 20573543]

15. Lesser RP, Kim SH, Beyderman L, Miglioretti DL, Webber WR, Bare M, et al. Brief bursts of pulse stimulation terminate afterdischarges caused by cortical stimulation. Neurology. 1999; 53:2073-2081. [PubMed: 10599784]

16. Motamedi GK, Lesser RP, Miglioretti DL, Mizuno-Matsumoto Y, Gordon B, Webber WR, et al. Optimizing parameters for terminating cortical afterdischarges with pulse stimulation. Epilepsia. 2002; 43:836-846. [PubMed: 12181002]

17. Engel J. Biomarkers in epilepsy: introduction. Biomark Med. 2011; 5:537-544. [PubMed: 22003902]

18. Worrell G, Gotman J. High-Frequency oscillations and other electrophysiological biomarkers of epilepsy: clinical studies. Biomark Med. 2011; 5:557-566. [PubMed: 22003904]

19. Bragin A, Engel J, Wilson CL, Fried I, Buzsáki G. High-frequency oscillations in human brain. Hippocampus. 1999; 9:137-142. [PubMed: 10226774]

20. Worrell GA, Parish L, Cranstoun SD, Jonas R, Baltuch G, Litt B. High-Frequency oscillations and seizure generation in neocortical epilepsy. Brain. 2004; 127:1496-1506. [PubMed: 15155522]

21. Schevon CA, Ng SK, Cappell J, Goodman RR, McKhann G, Waziri A, et al. Microphysiology of epileptiform activity in human neocortex. J Clin Neurophysiol. 2008; 25:321-330. [PubMed: 18997628]

22. Stead M, Bower M, Brinkmann BH, Lee K, Marsh WR, Meyer FB, et al. Microseizures and the spatiotemporal scales of human partial epilepsy. Brain. 2010; 133:2789-2797. [PubMed: 20685804] 
23. Buzsáki G, Silva FL. High frequency oscillations in the intact brain. Prog Neurobiol. 2012; 98:241-249. [PubMed: 22449727]

24. Worrell GA, Gardner AB, Stead SM, Hu S, Goerss S, Cascino GJ, et al. High-frequency oscillations in human temporal lobe: simultaneous microwire and clinical macroelectrode recordings. Brain. 2008; 131:928-937. [PubMed: 18263625]

25. Matsumoto A, Brinkmann BH, Stead SM, Matsumoto J, Kucewicz M, Marsh WR, et al. Pathological and physiological high-frequency oscillations in focal human epilepsy. $\mathrm{J}$ Neurophysiol. 2013; 110:1958-1964. [PubMed: 23926038]

26. Shelley, MW. Frankenstein, or the modern Prometheus. London: Lackington, Hughes, Harding, Mavor and Jones; 1818.

27. Selimbeyoglu A, Parvizi J. Electrical stimulation of the human brain: perceptual and behavioral phenomena reported in the old and new literature. Front Hum Neurosci. 2010; 4:46. [PubMed: 20577584]

28. Sanai N, Mirzadeh Z, Berger MS. Functional outcome after language mapping for glioma resection. N Engl J Med. 2008; 358:18-27. [PubMed: 18172171]

29. Nossek E, Korn A, Shahar T, Kanner AA, Yaffe H, Marcovici D, et al. Intraoperative mapping and monitoring of the corticospinal tracts with neurophysiological assessment and 3-dimensional ultrasonography-based navigation. J Neurosurg. 2011; 114:738-746. [PubMed: 20799862]

30. Haglund MM, Berger MS, Shamseldin M, Lettich E, Ojemann GA. Cortical localization of temporal lobe language sites in patients with gliomas. Neurosurgery. 1994; 34:567-576. [PubMed: 7516498]

31. Nathan SS, Sinha SR, Gordon B, Lesser RP, Thakor NV. Determination of current density distributions generated by electrical stimulation of the human cerebral cortex. Electroencephalogr Clin Neurophysiol. 1993; 86:183-192. [PubMed: 7680994]

32. Tolias AS, Sultan F, Augath M, Oeltermann A, Tehovnik EJ, Schiller PH, et al. Mapping cortical activity elicited with electrical microstimulation using FMRI in the macaque. Neuron. 2005; 48:901-911. [PubMed: 16364895]

33. Matsumoto R, Nair DR, LaPresto E, Bingaman W, Shibasaki H, Lüders HO. Functional connectivity in human cortical motor system: a cortico-cortical evoked potential study. Brain. 2007; 130:181-197. [PubMed: 17046857]

34. David O, Job AS, De Palma L, Hoffmann D, Minotti L, Kahane P. Probabilistic functional tractography of the human cortex. Neuroimage. 2013; 80:307-317. [PubMed: 23707583]

35. Roland J, Brunner P, Johnston J, Schalk G, Leuthardt EC. Passive real-time identification of speech and motor cortex during an awake craniotomy. Epilepsy Behav. 2010; 18:123-128. [PubMed: 20478745]

36. Korostenskaja M, Wilson AJ, Rose DF, Brunner P, Schalk G, Leach J, et al. Real-time functional mapping with electrocorticography in pediatric epilepsy: comparison with fMRI and ESM findings. Clin EEG Neurosci. 2013; 45:205-211. [PubMed: 24293161]

37. Prueckl R, Kapeller C, Potes C, Korostenskaja M, Schalk G, Lee KH, et al. CortiQ - clinical software for electrocorticographic real-time functional mapping of the eloquent cortex. Conf Proc IEEE Eng Med Biol Soc. 2013; 2013:6365-6368. [PubMed: 24111197]

38. Miller KJ, Leuthardt EC, Schalk G, Rao RP, Anderson NR, Moran DW, et al. Spectral changes in cortical surface potentials during motor movement. J Neurosci. 2007; 27:2424-2432. [PubMed: 17329441]

39. Manning JR, Jacobs J, Fried I, Kahana MJ. Broadband shifts in local field potential power spectra are correlated with single-neuron spiking in humans. J Neurosci. 2009; 29:13613-13620. [PubMed: 19864573]

40. Miller KJ. Broadband spectral change: evidence for a macroscale correlate of population firing rate? J Neurosci. 2010; 30:6477-6479. [PubMed: 20463210]

41. Miller KJ, Sorensen LB, Ojemann JG, den Nijs M. Power-law scaling in the brain surface electric potential. PLoS Comput Biol. 2009a; 5:e1000609. [PubMed: 20019800]

42. Miller KJ, Zanos S, Fetz EE, den Nijs M, Ojemann JG. Decoupling the cortical power spectrum reveals real-time representation of individual finger movements in humans. J Neurosci. 2009b; 29:3132-3137. [PubMed: 19279250] 
43. Miller KJ, Hermes D, Honey CJ, Hebb AO, Ramsey NF, Knight RT, et al. Human motor cortical activity is selectively phase-entrained on underlying rhythms. PLoS Comput Biol. 2012; 8:e1002655. [PubMed: 22969416]

44. Crone NE, Miglioretti DL, Gordon B, Sieracki JM, Wilson MT, Uematsu S, et al. Functional mapping of human sensorimotor cortex with electrocorticographic spectral analysis I Alpha and beta event-related desynchronization. Brain. 1998; 121:2271-2299. [PubMed: 9874480]

45. Miller KJ, Schalk G, Fetz EE, den Nijs M, Ojemann JG, Rao RP. Cortical activity during motor execution, motor imagery, and imagery-based online feedback. Proc Natl Acad Sci USA. 2010; 107:4430-4435. [PubMed: 20160084]

46. Hermes D, Miller KJ, Vansteensel MJ, Aarnoutse EJ, Leijten FS, Ramsey NF. Neurophysiologic correlates of fMRI in human motor cortex. Hum Brain Mapp. 2012; 33:1689-1699. [PubMed: 21692146]

47. Miller KJ, Abel TJ, Hebb AO, Ojemann JG. Reorganization of large-scale physiology in hand motor cortex following hemispheric stroke. Neurology. 2011; 76:927-929. [PubMed: 21383330]

48. Calvet AF, Bancaud J. Electrocorticography of waves associated with eye movements in man during wakefulness. Electroencephalogr Clin Neurophysiol. 1976; 40:457-469. [PubMed: 57034]

49. Fried I, Ojemann GA, Fetz EE. Language-related potentials specific to human language cortex. Science. 1981; 212:353-356. [PubMed: 7209537]

50. Hill NJ, Gupta D, Brunner P, Gunduz A, Adamo M, Ritaccio AL, et al. Recording human electrocorticographic (ECoG) signals for neuroscientific research and real-time functional cortical mapping. J Visual Exp. 2012; 64:e3993. http://www.jove.com/video/3993.

51. Wolpaw, JR.; Wolpaw, EW. Brain-Computer Interfaces. New York: Oxford University Press; 2012. p. 123-146.

52. Crone NE, Miglioretti DL, Gordon B, Lesser RP. Functional mapping of human sensorimotor cortex with electrocorticographic spectral analysis II Event-related synchronization in the gamma band. Brain. 1998; 121:2301-2315. [PubMed: 9874481]

53. Szczepanski SM, Parvizi J, Kuperman RA, Auguste KI, Crone NE, Knight RT. Dynamic changes in phase-amplitude coupling facilitate spatial attention control in fronto-parietal cortex. PLoS Biol. In press.

54. Axmacher N, Henseler MM, Jensen O, Weinreich I, Elger CE, Fell J. Cross-frequency coupling supports multi-item working memory in the human hippocampus. Proc Natl Acad Sci USA. 2010; 107:3228-3233. [PubMed: 20133762]

55. Durschmid S, Zaehle T, Kopitzki K, Schmitt FC, Voges J, Henze H, et al. Phase-amplitude crossfrequency coupling in the human nucleus accumbens tracks action monitoring during cognitive control. Front Hum Neurosci. 2013; 7:634. [PubMed: 24109447]

56. Durschmid S, Quandt F, Kramer U, Hinrichs H, Schulz R, Pannek H, et al. Oscillatory dynamics track motor learning in human cortex. PLoS One. 2014; 9:e89576. [PubMed: 24586885]

57. Jacobs J, Kahana MJ. Direct brain recordings fuel advances in cognitive electrophysiology. Trends Cogn Sci. 2010; 14:162-171. [PubMed: 20189441]

58. Jacobs J, Kahana MJ. Neural representations of individual stimuli revealed by gamma-band electrocorticographic activity. Journal of Neurosci. 2009; 29:10203-10214.

59. van Gerven MA, Maris E, Sperling M, Sharan A, Litt B, Anderson C, et al. Decoding the memorization of individual stimuli with direct human brain recordings. Neuroimage. 2013; 70:223-232. [PubMed: 23298746]

60. Jacobs J, Lega B, Anderson C. Explaining how brain stimulation can evoke memories. J Cogn Neurosci. 2012; 24:553-563. [PubMed: 22098266]

61. Penfield W. Some mechanisms of consciousness discovered during electrical stimulation of the brain. Proc Natl Acad Sci USA. 1958; 44:51-66. [PubMed: 16590173] 


\section{HIGHLIGHTS}

- ECoG can elucidate brain function in ways not achieved by other imaging modalities.

- ECoG-based applications are beginning to be used clinically.

- ECoG-based applications are beginning to be marketed commercially.

- Improvements continue in ECoG signal acquisition, analysis, and interpretation.

- ECoG is elucidating fundamental brain functions such as languge and memory. 
ECoG

\section{ECoG \& Brain Mapping}

100

50
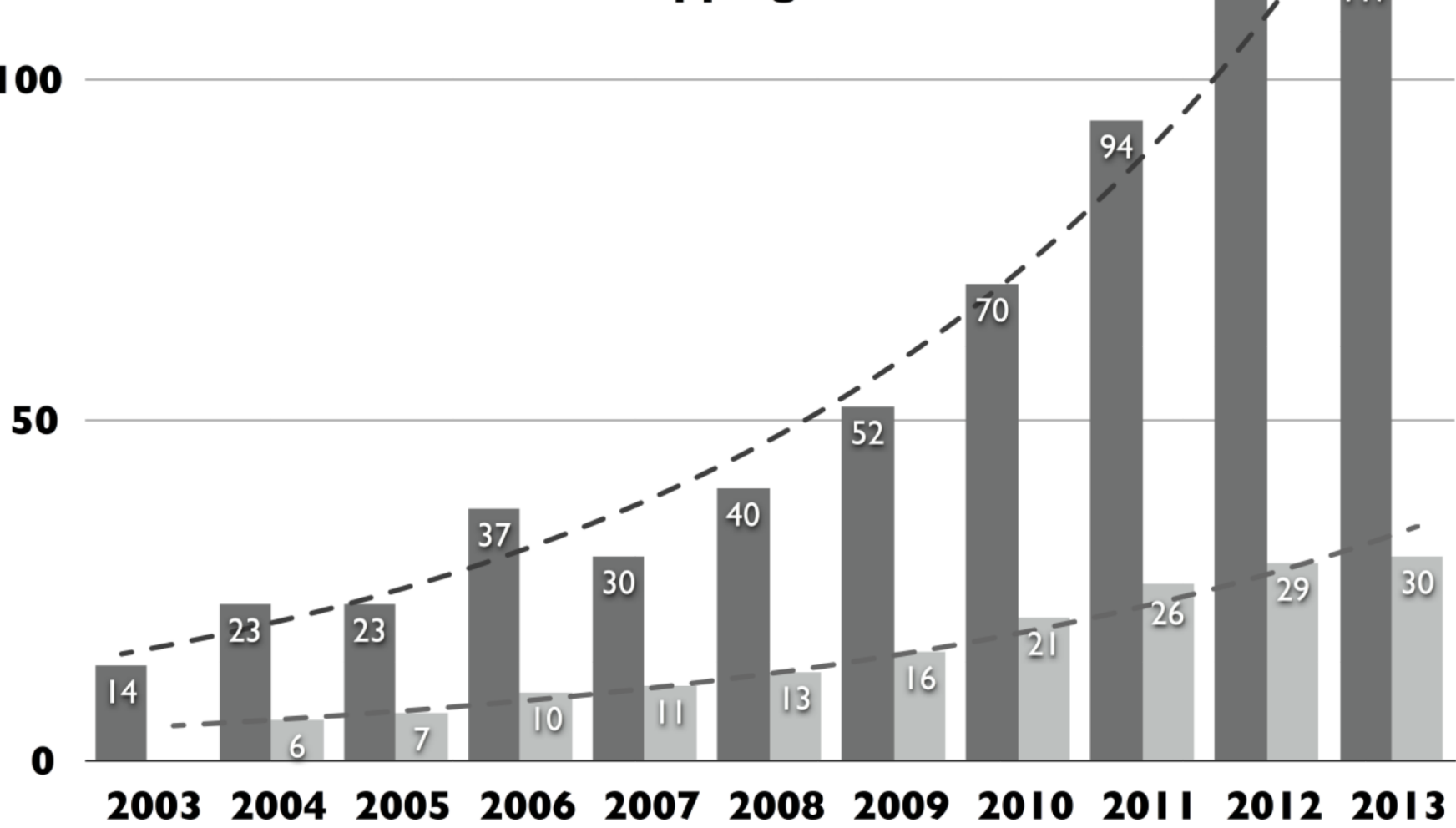

Fig. 1. Results of simple searches in PubMed for the terms "electrocorticography" and, separately, "electrocorticography and brain mapping"

Results demonstrate a roughly tenfold increase in publication output and investigators. 


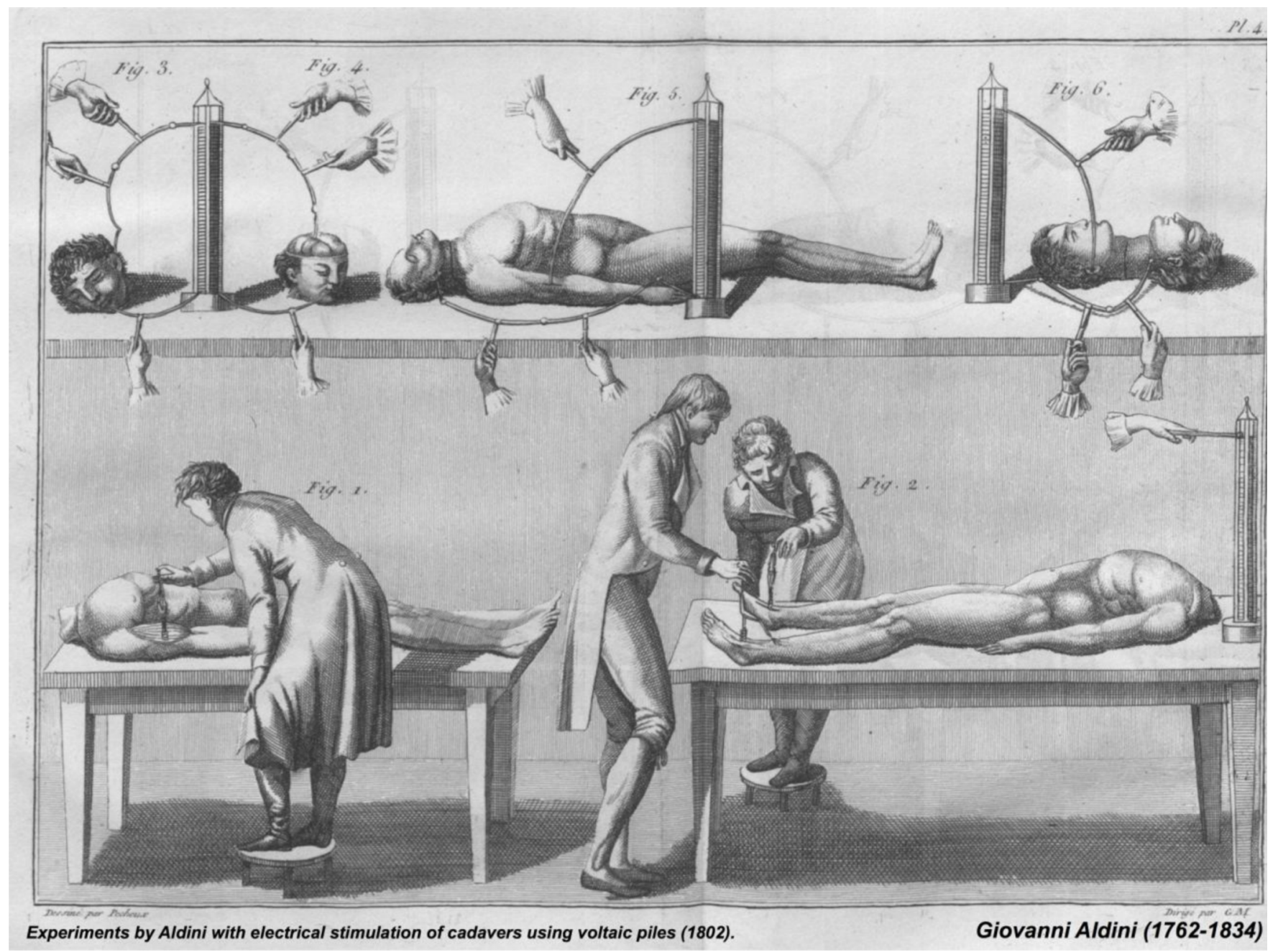

Fig. 2. Experiments by Giovanni Aldini (1762-1834) with electrical stimulation of cadavers using voltaic piles (1802) 

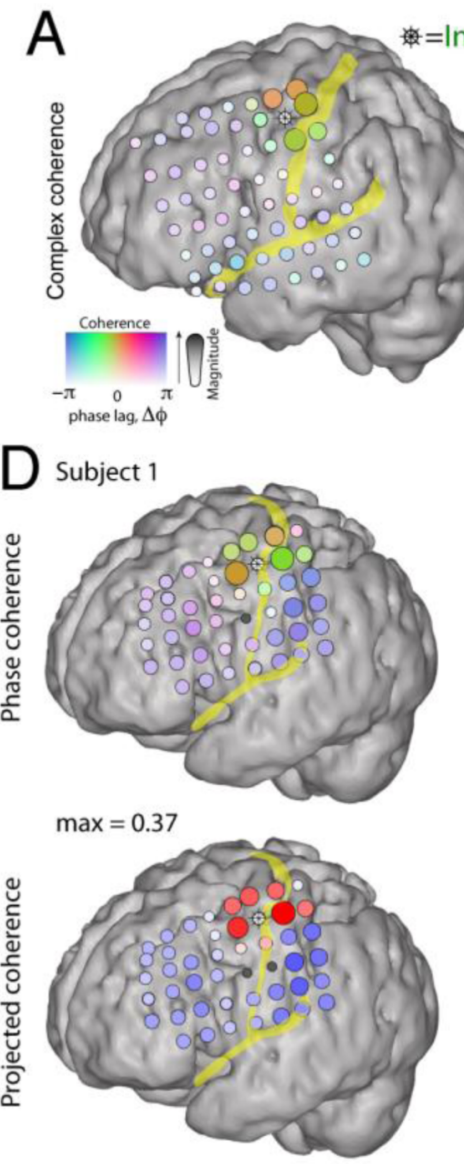

*Index site
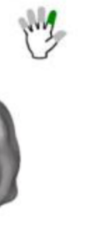

$\max =0.32$

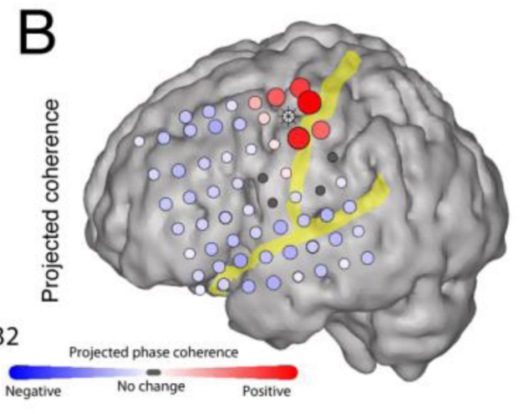

E subject 2
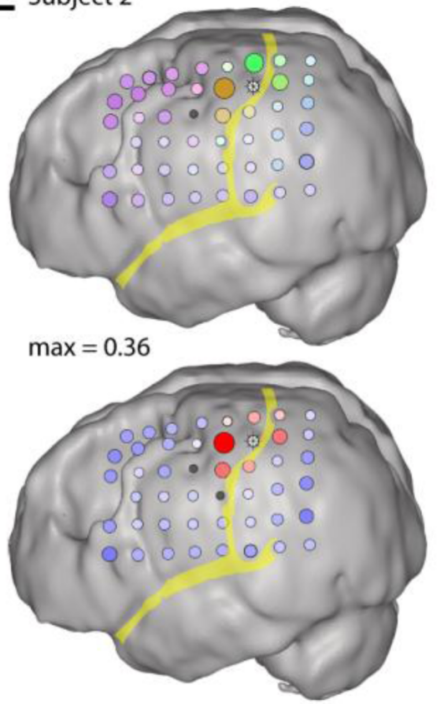
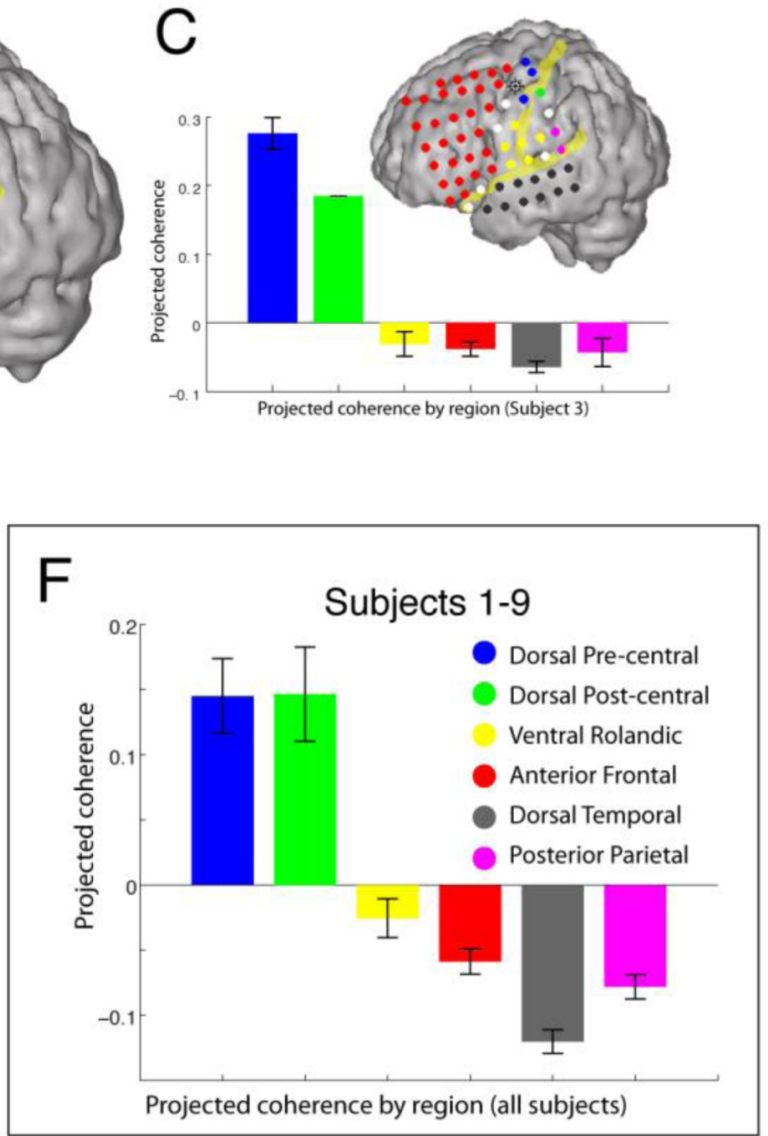

Fig. 3. Pairwise 12-20 Hz phase coherence with a pericentral motor cortex

A seed for the coherence measure is chosen by selecting the most index finger-specific site (identified by magnitude of broadband shift), denoted with "ship wheel" symbol. (A) The pairwise phase coherence between sites is calculated with the remainder of the array (during rest periods). The magnitude of the phase coherence ( $\max =0.32$ ) is reflected in the strength of the color and the electrode diameter, whereas the relative phase-lag of the phase coherence is denoted by the color. (B) To more clearly isolate spatial changes in phase, the complex phase coherence at each site was projected onto the phase of the site of maximum absolute phase coherence. (C) The mean projected phase coherence from each region (shown color coded on inset cortical rendering) is quantified, with error bars denoting the standard error of the mean within each region. (D and E) As in A and B; maximum phase coherence noted in between cortical renderings. (F) Pooled data from subjects $1-9$, showing that the $12-20 \mathrm{~Hz}$ pairwise phase coherence is conserved within dorsal pericentral cortex, bounded by the precentral sulcus anteriorly and the postcentral sulcus posteriorly. Note that the "anti-phase coherence" is most strongly due to introduced phase coherence in the common-average process ( $\pi$ out of phase) in the electrodes that do not otherwise have a large $\beta$-rhythm. See Miller et al. [42] for a complete description. 

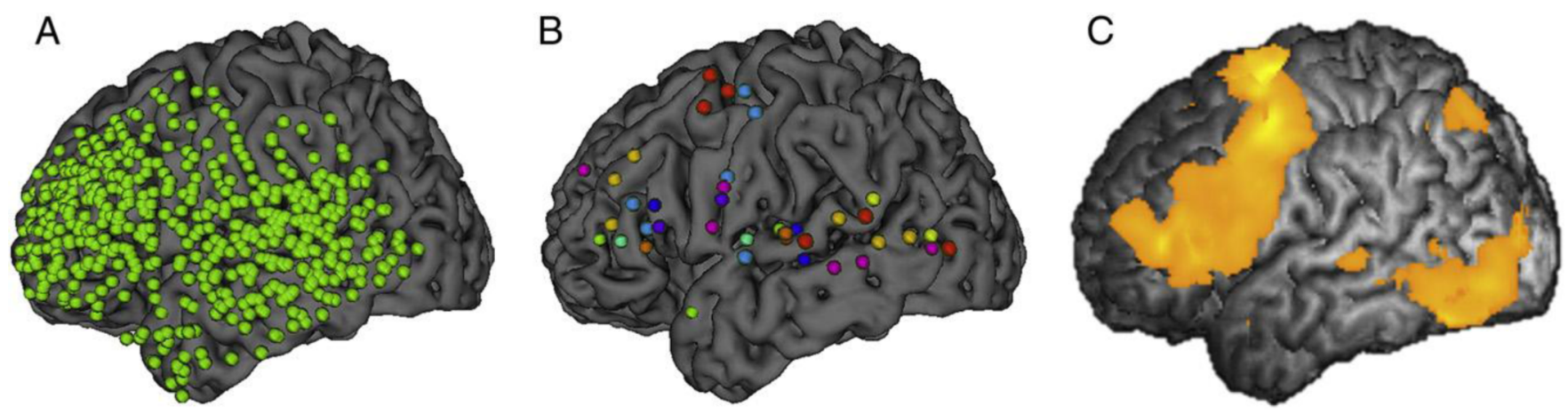

Fig. 4. Comparisons between distributions of HGAs and BOLD responses on a template brain (A) All ECoG electrodes displayed on a template brain. The electrodes (green dots) on the template brain widely covered the lateral aspect of the left frontal and temporal lobes. (B) ECoG electrodes with significant HGA. Different colors of the electrodes indicate individual patients. The electrodes were mainly clustered on the inferior frontal, superior and middle temporal gyri, and precentral gyrus (premotor cortex and face-motor area). (C) A threedimensional t-map of BOLD response across individual displayed on a template brain. The BOLD responses were widely observed in the frontal lobe, which involves the inferior frontal and precentral gyri. There were additional activated areas in the inferior temporal gyrus, which was sparsely covered by ECoG electrodes. Time-frequency analysis was applied to compare their spatiotemporal profiles. 


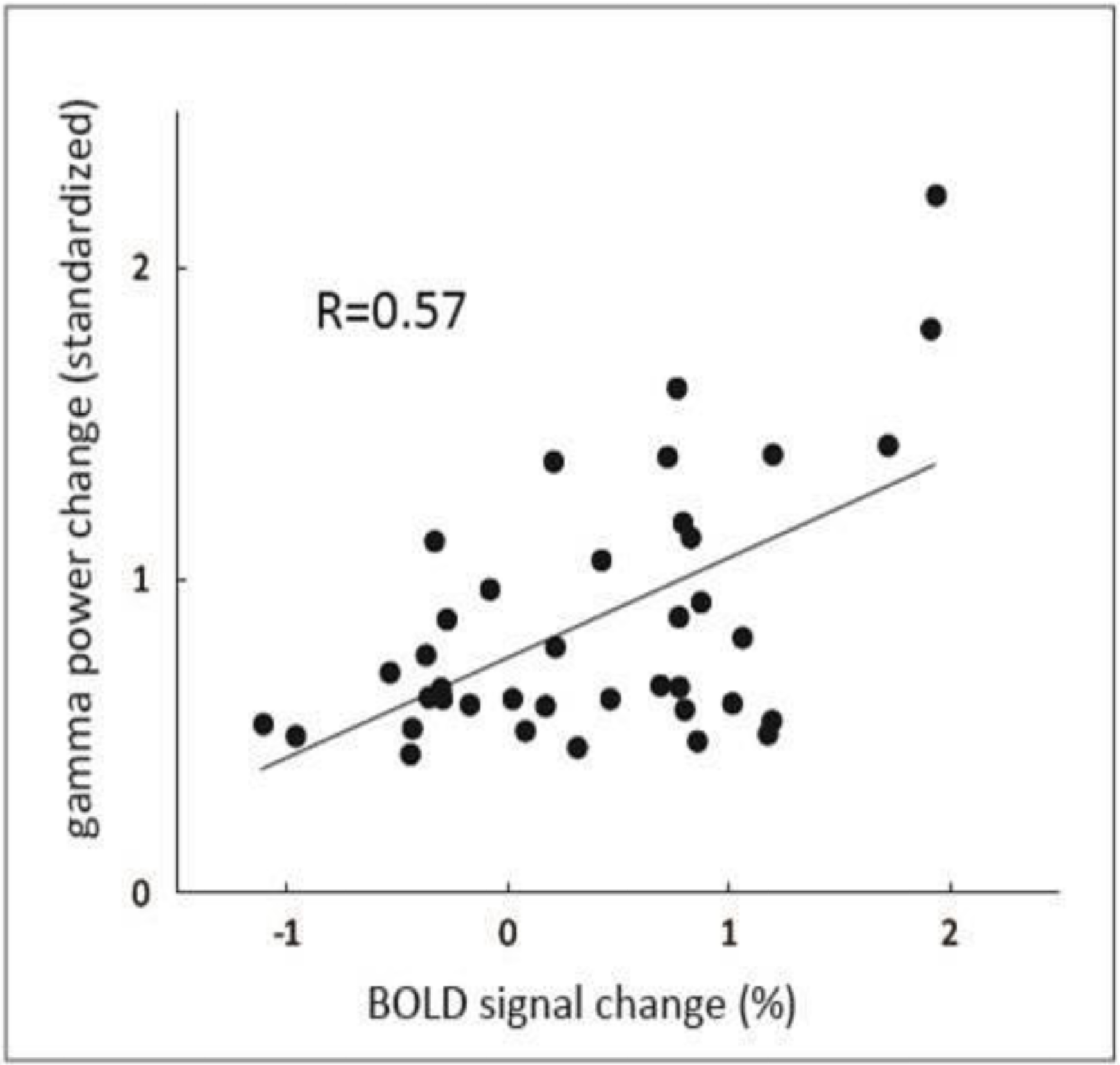

Fig. 5. High gamma activity (HGA) vs. blood oxygen level-dependent (BOLD) imaging signal change

Comparison of normalized HGA and BOLD signal show a positive correlation $(R=0.57, \mathrm{p}$ $=0.0002$ ) between these two assessment tools. 

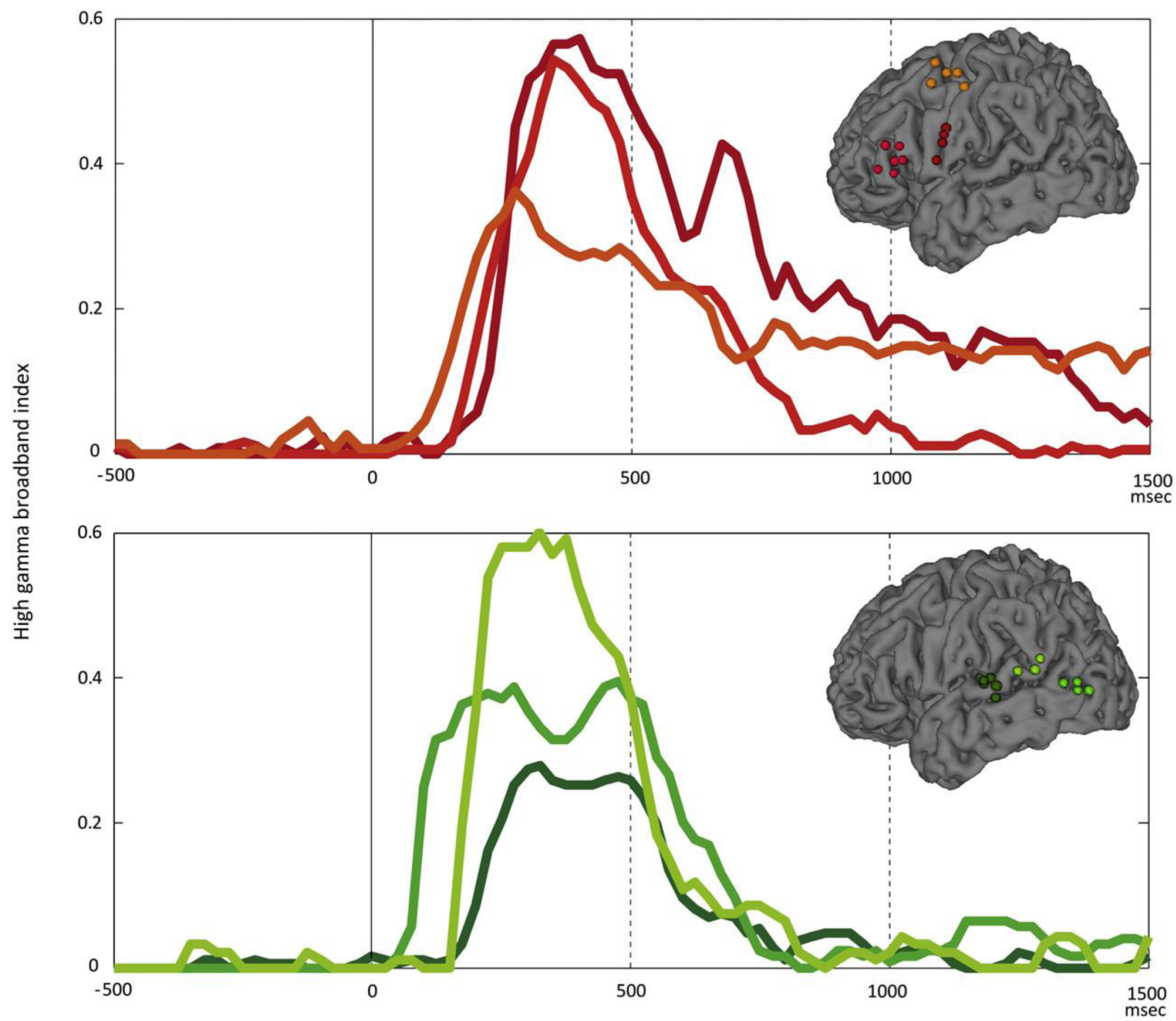

Fig. 6. Temporal dynamics of high gamma activity of noticeable electrode clusters in frontal and temporal lobes

Each line color corresponds to electrode color. $\mathrm{X}$ and $\mathrm{Y}$ axes indicate latency and high gamma broadband index (HGBI), respectively. HGBI in the frontal lobe lasts longer beyond $1000 \mathrm{msec}$. On the other hand, rapid decline of HGBI was observed after $500 \mathrm{msec}$ in the temporal lobe. 
A

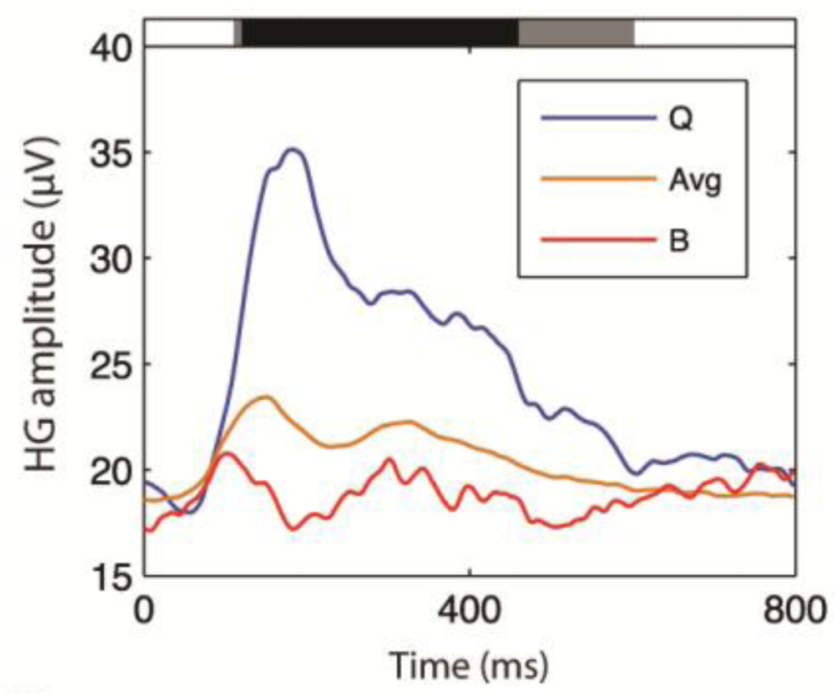

C

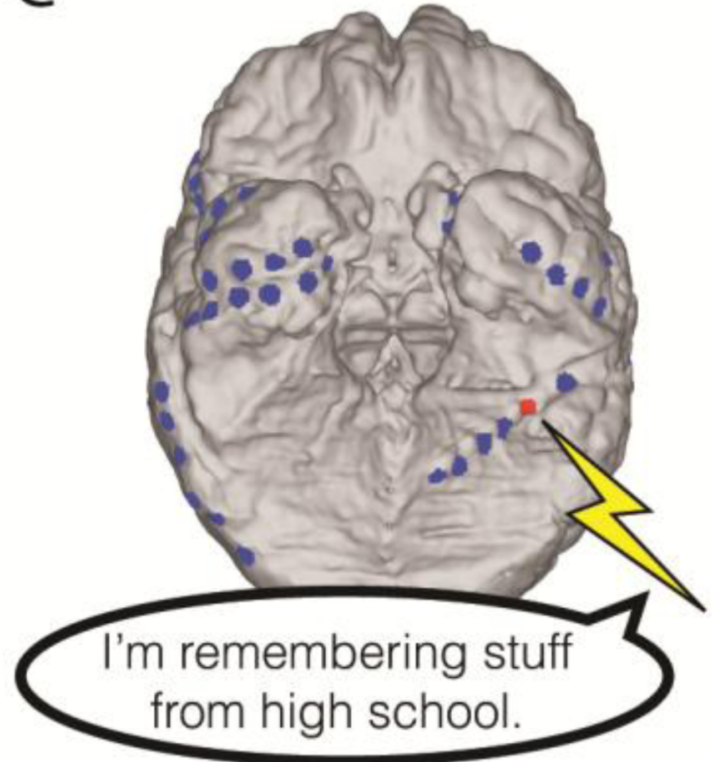

B

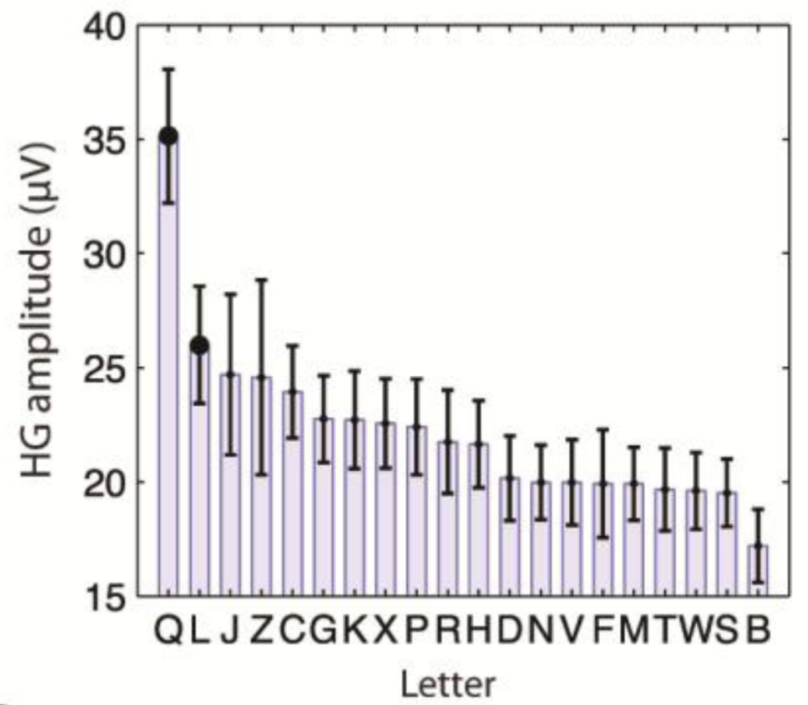

D

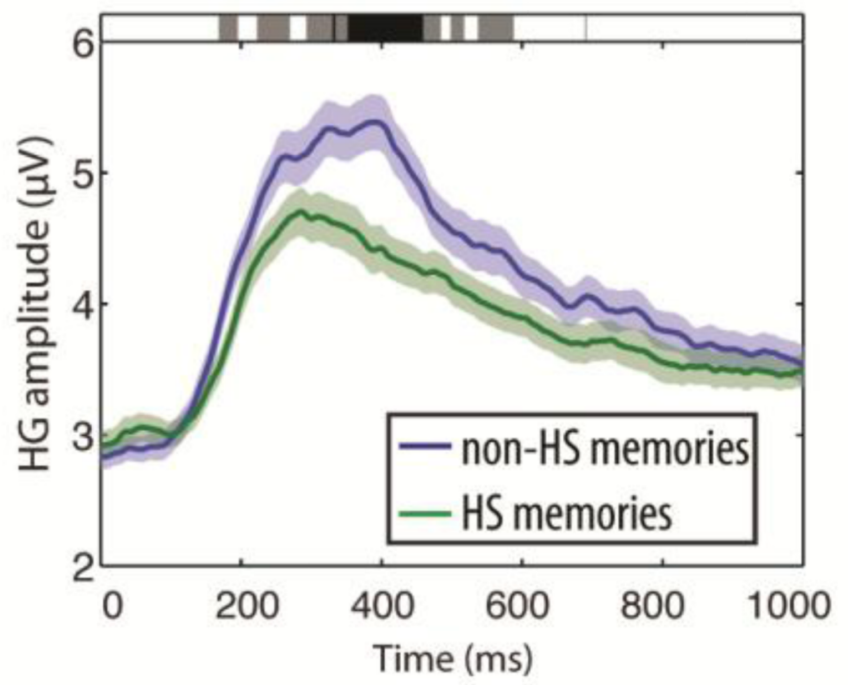

Fig. 7. Electrocorticography reveals the neural basis of memories

(A) The amplitude of high gamma (HG; 65-150 Hz) ECoG activity at a site in one patient's ventral temporal lobe in a working memory task (Modified from Jacobs and Kahana 2009). Shading at top indicates time points that exhibited significant differences in amplitude according to the identity of the viewed item. (B) The amplitude of HG activity for all letters viewed in the task (same electrode as panel A.) (C) Brain image from a patient who spontaneously remembered memories of high school (HS) after stimulation at the indicated electrode (red) in his left ventral temporal lobe. (D) The amplitude of HG activity observed from this electrode when the patient performed a memory task where he remembered HS and non-HS information (same electrode as panel C.) 